\title{
Development and in Vitro Characterization of Photochemically Crosslinked Polyvinylpyrrolidone Coatings for Drug-Coated Balloons
}

\author{
Svea Petersen ${ }^{1}{ }^{*}$, Ingo Minrath ${ }^{1}$, Sebastian Kaule ${ }^{1}$, Jürgen Köcher ${ }^{2}$, Klaus-Peter Schmitz ${ }^{1}$ \\ and Katrin Sternberg ${ }^{1}$
}

1 Institute for Biomedical Engineering, University of Rostock, Friedrich-Barnewitz-Straße 4, 18119 Rostock, Germany; E-Mails: ingo.minrath@uni-rostock.de (I.M.); sebastian.kaule@uni-rostock.de (S.K.); klaus-peter.schmitz@uni-rostock.de (K.-P.S.), katrin.sternberg@uni-rostock.de (K.S.)

2 Bayer Material Science AG, BMS-CAS-INN, Leverkusen, Q 1, 212, Germany; E-Mail: juergen.koecher@bayer.com

* Author to whom correspondence should be addressed; E-Mail: svea.petersen@uni-rostock.de; Tel.: +49-381-54345-531; Fax: +49-381-54345-502.

Received: 20 September 2013; in revised form: 7 November 2013 / Accepted: 27 November 2013 / Published: 5 December 2013

\begin{abstract}
Polyvinylpyrrolidone (PVP) is a conventionally applied hydrophilic lubricious coating on catheter-based cardiovascular devices, used in order to ease movement through the vasculature. Its use as drug reservoir and transfer agent on drug-coated balloons (DCB) is therefore extremely promising with regard to the simplification of its approval as a medical device. Here, we developed a PVP-based coating for DCB, containing paclitaxel (PTX) as a model drug, and studied the impact of crosslinking via UV radiation on drug stability, wash off, and transfer during simulated use in an in vitro vessel model. We showed that crosslinking was essential for coating stability and needed to be performed prior to PTX incorporation due to decreased drug bioavailability as a result of photodecomposition and/or involvement in vinylic polymerization with PVP under UV radiation. Moreover, the crosslinking time needed to be carefully controlled. While short radiation times did not provide enough coating stability, associated with high wash off rates during DCB insertion, long radiation times lowered drug transfer efficiency upon balloon expansion. A ten minutes radiation of PVP, however, combined a minimized drug wash off rate of $34 \%$ with an
\end{abstract}


efficient drug transfer of $49 \%$, underlining the high potential of photochemically crosslinked PVP as a coating matrix for DCB.

Keywords: implant coating; drug-coated balloons; hydrogel; drug delivery; crosslinking

\section{Introduction}

The use of percutaneous, catheter-based vascular devices to treat coronary and peripheral artery disease, caused by arteriosclerosis, has become the standard of care. In order to overcome associated abrupt vessel re-narrowing or closure, antiproliferative drugs are often additionally administered during therapy by the application of well-known drug-eluting stents (DES) or drug-coated balloons (DCB) [1,2]. Major potential advantages of DCB in comparison to DES include the absence of a remaining foreign body in the artery, high initial drug delivery with little impact on long-term healing possibly limiting the risk of late stent thrombosis, and high deliverability, opening the opportunity of use in small vessels, bifurcations, long lesions, etc., hardly accessible by stents [3,4]. While the drug of choice on currently investigated DCB is paclitaxel (PTX), which has proven rapid uptake by the intima, high retention rate, and sustained biological effect [5], various types of surface designs are discussed. As PTX alone is very lipophilic and, hence, sticks to the balloon surface, transfer agents, as the mainly studied contrast agent iopromide [6], but also urea [7] and plasticizers [8], are applied in order to enhance the drug transfer capability. These are generally mixed in a varying content with the drug in an applicable solvent, and the resulting solutions/suspensions are deposited to the balloon surface by means of one of the numerous established coating approaches for vascular implants, mainly micropipetting, dip-coating, and spray-coating [9]. In contrast to DES coating designs, which mostly involve hydrophobic polymers as a drug reservoir in order to allow a sustained drug release over a long time period [10], described coating matrices used for DCB are rather hydrophilic and loose to guarantee spontaneous drug transfer upon balloon expansion. Therefore, drug wash off rates during transit of the device through the vascular system should not be underestimated. For instance, while Kelsch et al. [11] reported percental PTX wash off rates of up to $26 \%$ and $36 \%$ for urea- or iopromide-based DCB, respectively, Berg et al. [12] observed a drug wash off rates of $42 \%$ for an iopromide-based formulation, both during in vitro passage through a hemostatic valve and a guiding catheter.

Having in mind that many of these interventional cardiovascular devices already incorporate a hydrophilic lubricious coating in order to ease movement through the vasculature [13,14], the use of the same hydrophilic coating as drug reservoir and transfer agent would be of high interest for DCB. When exposed to aqueous environments, these lubricious coatings may absorb a multiple of times its dry weight in water. One can, hence, presume that a previously embedded lipophilic drug as PTX will be protected from elution until the coating is compressed leading to simultaneous release of water and drug. However, formation of such hydrogels with this above described swelling behavior affords controlled crosslinking. The most commonly used lubricious coatings in medical devices consist of water-soluble polymers, such as polyvinylpyrrolidone (PVP) [15], which is approved by FDA, as a biocompatible and non-antigenic compound and widely used in pharmaceutical formulations [16]. Crosslinking of PVP can be performed via chemical reactions, e.g., Fentons reaction relying on the reduction of hydrogen 
peroxide at the expense of $\mathrm{Fe}^{2+}$-ions under formation of hydroxyl radicals initiating vinylic polymerization [17]. Due to the non-necessity of chemical reagents with the advantage of improved biocompatibility, electron beam, or gamma radiation, which similarly lead to hydroxyl radicals from water radiolysis, are more convenient for medical devices [18]. However, by the use of these high-energy radiative processes, degradation of polymeric main chains might compete with successful PVP crosslinking [19]. Therefore, ultraviolet (UV) radiation presents an inexpensive alternative for PVP hydrogel formation, which involves photoreactions as $\beta$-cleavage, inter- and intramolecular photoreduction, photorearrangements, etc., of the pyrrolidone substituent, intermediated by crosslinking-initializing radicals [20].

With these reasons, the objective of this study is to develop a PTX containing, PVP-based coating for DCB and to study the impact of crosslinking via UV radiation on drug stability, wash off, and transfer during simulated use in an in vitro vessel model. In this context, we focus on the improvement of DCB with regard to efficient drug delivery using coating components already conventionally applied on catheter-based vascular devices. Additionally, we aim at the provision of in vitro data on the performance of DCB, which is, to date, rarely found in literature.

\section{Experimental Section}

\subsection{Materials}

All chemicals were purchased from Sigma-Aldrich (Taufkirchen, Germany), Mallinckrodt Baker (Griesheim, Germany), SERVA Feinbiochemica (Heidelberg, Germany), Thermo Scientific (Karlsruhe, Germany), or Merck (Darmstadt, Germany) in pro analysi quality or higher, unless indicated differently. PVP (K90, $M_{\mathrm{w}}=360,000 \mathrm{~g} / \mathrm{mol}$, Sigma-Aldrich, Taufkirchen, Germany) and PTX (Cfm Oskar Tropitzsch e.K., Marktredwitz, Germany) were used as coating and model drug, respectively. For the estimation of crosslinking efficiency with regard to PVP wash off, swelling in aqueous media, and the influence of crosslinking on drug stability, glass cover slips $(\phi=9 \mathrm{~mm}$, VWR International, Darmstadt, Germany) were used as coating carrier. For all simulated use experiments, uncoated balloon catheters of $4 \mathrm{~mm}$ in diameter and $20 \mathrm{~mm}$ in length were kindly provided by Biotronik SE and Co. KG (Erlangen, Germany).

\subsection{Coating}

\subsubsection{Coating of Glass Cover Slips with PVP and/or PTX}

For the sake of convenience, glass cover slips were coated with PVP and/or PTX via a pipetting process. Therefore, PVP was dissolved in chloroform to yield a concentration of $15.4 \mathrm{mg} / \mathrm{mL}$. If indicated, PTX was additionally added to obtain a final PTX concentration of $25 \%$ in PVP $(w / w)$. For a pure PTX coating, PTX was dissolved in chloroform to yield a concentration of $5.1 \mathrm{mg} / \mathrm{mL}$. Thirty-seven microliters of one of the resulting solutions were then slowly pipetted, for each glass slide $\left(64 \mathrm{~mm}^{2}\right)$ in order to approach a surface load of $9 \mu \mathrm{g} / \mathrm{mm}^{2}$ PVP and/or $3 \mu \mathrm{g} / \mathrm{mm}^{2}$ PTX. This PTX load has been defined, as it had been shown to be safe and effective in non-clinical [21] and clinical studies [22,23]. Prior to crosslinking, all coatings were dried in a vacuum chamber at $40{ }^{\circ} \mathrm{C}$ and $40 \mathrm{mbar}$ 
overnight. Coating mass control was performed by weighing glass slides before and after the coating process using a microbalance (UMX5, Mettler Toledo, Giessen, Germany).

\subsubsection{Coating of Balloon Catheters}

For improved hydrogel covering on the more complex geometry of balloon catheters, a PVP spray-coating process was established. Therefore, PVP was dissolved in chloroform to yield a concentration of $0.25 \%(w / w)$. If indicated, PTX was additionally added to obtain a final PTX concentration of $25 \%$ in PVP $(w / w)$. Prior to coating, balloons were dilated via a manual pump ( $p=2$ bar). For spraying, balloon catheters were inserted into a holder of an electropneumatic airbrush system, which guaranteed homogeneous coating by continuous rotation along their longitudinal axis. By means of coating-intermediate weighing using a microbalance, we determined the spraying time needed for the deposition of total coating masses of, $2260 \mu \mathrm{g}$ without, and $3010 \mu \mathrm{g}$ with $3 \mu \mathrm{g} / \mathrm{mm}^{2}$ PTX incorporation, respectively. Prior to crosslinking, all coatings were dried in a vacuum chamber at $40{ }^{\circ} \mathrm{C}$ and 40 mbar overnight.

\subsubsection{Crosslinking Conditions}

Crosslinking of PVP coatings was performed by irradiations using a 3UV lamp (Ultra Violet Products, P/N 95-0343-02, 254-302-365 nm, 8 W/230 V, 50 Hz/0.16 A, Cambridge, UK) after drying and prior to PTX incorporation via pipetting. Therefore, coated cover slips or balloon catheters were placed beneath the lamp in a distance of $15 \mathrm{~mm}$. The lamp of $8 \mathrm{~W}$ was set to $254 \mathrm{~nm}$, which produces a radiant flux of $1670 \mu \mathrm{W} / \mathrm{cm}^{2}$ at a $2^{\prime \prime}$ distance. Higher wavelengths $(302 \mathrm{~nm}, 365 \mathrm{~nm}$ ) resulted in inefficient crosslinking, as evidenced by coating stability (data not shown). Balloon catheters were rotated intermediately during the crosslinking process.

\subsubsection{Incorporation of PTX via Pipetting}

Unless indicated differently, PTX incorporation was performed via pipetting after the irradiation process in order to guarantee a consistent surface load of $3 \mu \mathrm{g} / \mathrm{mm}^{2}$. Therefore, PTX was dissolved in ethanol $(\mathrm{EtOH}) / \mathrm{dH}_{2} \mathrm{O}(8 / 2(v / v))$ mixture to yield a concentration of $7.54 \mathrm{mg} / \mathrm{mL}$. One-hundred microliters of this solution were then slowly pipetted per balloon catheter under rotation in a weak air stream. The volume of $100 \mu \mathrm{L}$ turned out to be best manageable for balloon catheters of the chosen dimension, while the solvent mixture has been chosen as it provides good PTX stability and enough swelling of the hydrogel allowing incorporation of the drug (data not shown). Finally, all coatings were dried in a vacuum chamber at $40{ }^{\circ} \mathrm{C}$ and 40 mbar overnight. Balloons were deflated by means of a manual pump, except those for characterization by scanning electron microscopy.

\subsection{Characterization}

\subsubsection{Scanning Electron Microscopy}

Examination of the coating morphology was carried out in a Philips XL 30 ESEM (Philips Electron Optics, Eindhoven, The Netherlands) operating in the ESEM mode with a water vapor pressure of 
1.2 mbar. The accelerating voltage was fixed to $10 \mathrm{kV}$ and the beam current to $11 \mu \mathrm{A}$. Working distance was adapted to each sample and varied from 14.3 to $30.8 \mathrm{~mm}$, indicated as WD in the legend of each micrograph. Samples were attached to the specimen mount, as obtained after the coating process, and examined at four different positions. Representative micrographs are shown.

\subsubsection{Coating Stability after Crosslinking}

In order to determine the stability of PVP coatings in function of crosslinking, coated glass slides were immersed for 1 or $2 \mathrm{~min}$ in $1 \mathrm{~mL}$ phosphate buffered saline (Dulbecco's PBS, pH 7.2) containing $0.06 \%(w / v)$ polyoxyethylene (20) sorbitan monolaurate (DPBS/Tween20). After drying in a vacuum chamber at $40{ }^{\circ} \mathrm{C}$ and 40 mbar overnight, the remaining coating mass was determined by means of a microbalance and compared to the initial coating mass.

\subsubsection{Oven-Based Karl Fischer (KF) Titration}

Before the KF measurements were conducted, PVP-coated glass slides were contacted for 1 or 2 min with $1 \mathrm{~mL}$ DPBS/Tween 20 and, thereafter, carefully dried to remove water droplets from the surface. Samples were subsequently placed in the sample holder of the Drying Oven D0308 (Mettler Toledo, Gießen, Germany) and heated up to $230{ }^{\circ} \mathrm{C}$ in an argon atmosphere. The water content was finally determined using a Karl-Fischer Coulometer C20 (Mettler Toledo, Gießen, Germany) with the Hydranal-Coulomat AG KF reagent (Sigma-Aldrich, St. Louis, MO, USA). All measurements were performed in four replicates. The water uptake was calculated as the difference in the water content of samples contacted with the aqueous solutions and the non-contacted as prepared samples.

\subsubsection{PTX Recovery after Crosslinking}

After crosslinking, glass slides were extracted for 30 min with $2 \mathrm{~mL}$ methanol $(\mathrm{MeOH})$ at $23 \pm 2{ }^{\circ} \mathrm{C}$. Then, the drug content was determined by means of HPLC after 1:10 dilution with $\mathrm{MeOH}$ using conditions described in the corresponding section and compared to the initial drug load.

\subsubsection{PTX Wash off, Transfer, Residual and Total Load}

PTX wash off, transfer, residual, and total load of the balloon catheters were assessed by means of HPLC using conditions described in the section HPLC parameter. PTX wash off refers to PTX elution, as occurring during balloon insertion. Therefore, balloons were firstly immersed for $1 \mathrm{~min}$ in $20 \mathrm{~mL}$ of DPBS/Tween 20 at $23 \pm 2{ }^{\circ} \mathrm{C}$. In a second step, the balloons were dilated for $30 \mathrm{~s}$ to 7 bar in a silicone tube (outer diameter: $4.1 \mathrm{~mm}$, inner diameter: $2.7 \mathrm{~mm}$ ) in $20 \mathrm{~mL}$ DPBS/Tween 20. Prior to HPLC measurement, samples were diluted 1:1 with $\mathrm{MeOH}$. Drug residue on the silicone tube and on the balloon was examined afterwards by PTX extraction in $3 \mathrm{~mL}$ and $20 \mathrm{~mL} \mathrm{MeOH}$, respectively, for $30 \mathrm{~min}$ at $23 \pm 2{ }^{\circ} \mathrm{C}$. Stated values for the transfer of PTX in the silicone tube present the sum of the PTX amount found in DPBS/Tween20 during dilatation and in the $\mathrm{MeOH}$ extract of the silicone tube. Total drug load represents the sum of all measured PTX amounts. 


\subsubsection{Simulated Use}

The process of coronary balloon angioplasty was simulated using an in vitro model, recently described in literature [24], consisting of a guiding catheter (Cordis Brite Tip; 5F; $100 \mathrm{~cm} ; 1.4 \mathrm{~mm}$ ID) with a guide wire (Biotronik Galeo M014) and a tortuous path revetted with a polytetrafluoroethylene (PTFE) tube. The anatomic model was equivalent to the model described in American Society for Testing and Materials (ASTM) F2394-07, X.2.4 (PM-40/2010) surrounded by $37 \pm 2{ }^{\circ} \mathrm{C}$ heated water. The connection between the guiding catheter and the inner PTFE tubing of the vessel model was sealed to avoid fluid loss or contamination of the inner lumen of the test path. On the distal end of the test path a silicone test tube ( $2.7 \mathrm{~mm}$ ID) was placed as a model of the stenotic vessel. After balloon deflation, if necessary, by means of a hand pump, all test samples were manually advanced over the guide wire through the guiding catheter and the test path of the model until the balloon was placed in the silicone tube. The guiding catheter and vessel model were then flushed repeatedly with in total $30 \mathrm{~mL}$ of $0.9 \%$ $\mathrm{NaCl}$ to recover particles and PTX released during tracking ("after track 1"). Afterwards, the balloon was dilated to 7 bar and held for $30 \mathrm{~s}$. After deflation of the balloon, the solution contained in the silicone tube was collected and filled up to $30 \mathrm{~mL}$ for particle and drug analysis ("after dilatation 1"), while the silicone tube was removed from the vessel model and stored in an empty flask. The balloon was cut and also stored in an empty flask for morphology assessment and residual drug content determination. The entire test path was then finally flushed once with $30 \mathrm{~mL} \mathrm{MeOH}$ for determination of the residual PTX content released during tracking ("after track 2") and, afterwards, with $0.9 \% \mathrm{NaCl}$ in preparation of the next test.

\subsubsection{Analysis of PTX Wash off and Transfer during Simulated Use}

The cut balloon and the silicone tube were first extracted for 30 min with $20 \mathrm{~mL}$ of $\mathrm{MeOH}$ at $23 \pm 2{ }^{\circ} \mathrm{C}$ ("residual balloon drug load", "after dilatation 2"). Then, the drug content in all collected solutions ("after track 1", "after track 2", "after dilatation 1", "after dilatation 2", and "residual balloon drug load") was determined by means of HPLC after 1:10 dilution with MeOH using conditions described in the following section. The determined drug content in the collected solutions after track 1 and 2, as well as in the solutions after dilatation 1 and 2, are summed up and presented in the results as drug wash off during track and drug transfer, respectively. The calculated total drug load is the sum of all measured PTX amounts.

\subsubsection{HPLC Parameter}

Twenty microliters of the test solutions were injected into an Eurospher column 100-5, C18, $120 \mathrm{~mm} \times 4 \mathrm{~mm}$ ID (Wissenschaftlicher Gerätebau Dr. -Ing. Herbert Knauer GmbH, Berlin, Germany). The chromatographic conditions were: column temperature $23{ }^{\circ} \mathrm{C}$, isocratic eluent PBS $(0.005 \mathrm{M}$, $\mathrm{pH} 3.5$ ) - acetonitrile $50-50 \%(\mathrm{v} / \mathrm{v})$, flow rate $1.0 \mathrm{~mL} / \mathrm{min}$ and $\mathrm{UV}$ detection at $230 \mathrm{~nm}$ with calibrated measurement range $0.5-20.0 \mathrm{mg} / \mathrm{L}$ and detection limit approximately $0.05 \mathrm{mg} / \mathrm{L}$. 


\section{Results and Discussion}

\subsection{Development and in Vitro Characterization of PVP-Coated DCB in Dependence of Crosslinking Conditions}

In order to estimate the potential of PVP on DCB, we coated bare balloons with PVP and PTX via a spray-coating process, based on two-component jet atomization technology. Investigation of the resulting DCB, with regard to PTX wash off and transfer upon balloon expansion in a silicone tube as a vessel model, evidences a wash off rate of nearly 50\% during 1 min immersion in DPBS/Tween20 and a transfer of approximately $25 \%$ of the total PTX load (Figure 1).

Figure 1. Absolute PTX wash off per $\mathrm{mm}^{2}$ balloon surface after 1 min immersion in DPBS/Tween20, PTX transfer upon expansion in silicone tube (30 s, 7 bar), residual and total PTX load of one DCB spray-coated with PVP containing 25 wt $\%$ PTX at $23 \pm 2{ }^{\circ} \mathrm{C}$.

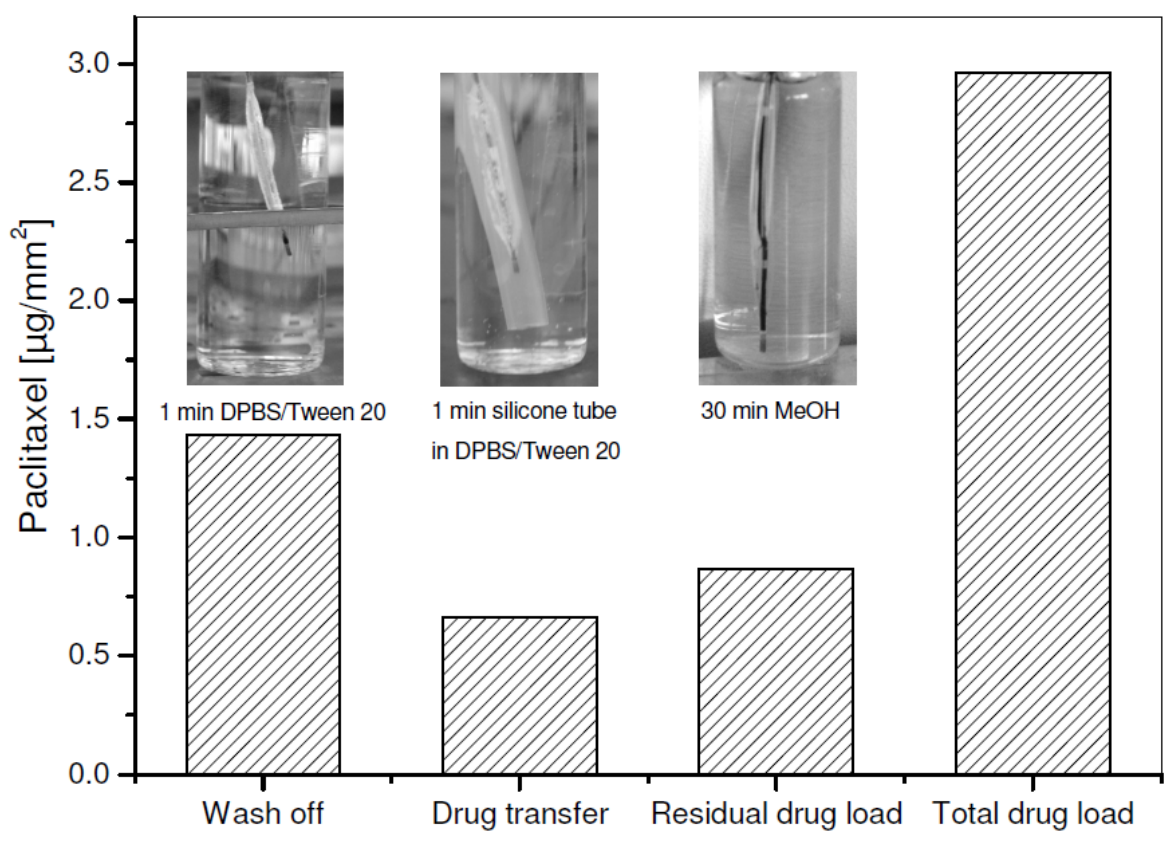

The application of the water-soluble PVP does hence not provide enough stability such that sufficient PTX will reach the vessel area to be treated. It has to be mentioned, that we have not simulated the passage of the DCB through the tortuous path of the coronary arteries at this time. Mechanical strain on the coating and drug wash off is consequently estimated higher under conditions in clinical routine. Crosslinking, in order to reduce the water solubility of PVP and possibly increase coating stability, seems, hence, necessary for the generation of good-performing DCB.

Crosslinking of PVP can be achieved via several chemical and photochemical reactions. With the purpose to establish an inexpensive and biocompatible coating method, we investigated a simple UV-radiation process. However, it is well-known that drugs are prone to photodecomposition, as contained molecular features, as for instance carbonyl groups or $\mathrm{C}=\mathrm{C}$ double bonds, are expected to induce photoreactivity [25]. In this context, we evaluated the influence of UV radiation time on PTX stability by the use of glass slides, coated with $3 \mu \mathrm{g} / \mathrm{mm}^{2}$ PTX, either embedded in $75 \mathrm{wt} \%$ PVP or free from matrix. Under radiation with $254 \mathrm{~nm}$, both coatings, with and without PVP, suffered from a loss of 
measurable PTX, while the decay was even faster in the presence of PVP (Figure 2). We dedicate this observation to the formation of radicals within the PTX molecule. The insert in Figure 2 proposes a possible radical formation mechanism involving excitation of the carbonyl group with or without rearrangement by cyclization and successive hydrogen transfer according to Chen et al. [26].

These radicals can either involve in vinylic polymerization with PVP and/or lead to decomposition of the drug. In both cases, PTX is not bioavailable anymore and possibly inactive with regard to the envisaged clinical application. It is, hence, necessary to integrate the PTX within the PVP coating after the crosslinking process by, for instance, an incorporation process as a pipetting process in order to guarantee a consistent PTX surface load of $3 \mu \mathrm{g} / \mathrm{mm}^{2}$, which has been shown to be safe and effective in non-clinical [21] and clinical studies [22,23]. The total DCB preparation process consequently involved (i) application of PVP via spray-coating, (ii) crosslinking of the deposited PVP coating via UV radiation at $254 \mathrm{~nm}$, and (iii) incorporation of PTX via pipetting, which are all well-described and applied coating approaches for DCB [9]. Representative ESEM micrographs of DCB, coated via this strategy, reveal the successful integration of PTX crystals within the crosslinked hydrogel matrix (Figure 3). This seems essential for reduction of PTX wash off as we observed in a previous study that the presence of free PTX crystals above the coating matrix resulted in high drug wash off rates [27].

Figure 2. Loss of free PTX $\left(\mathrm{PTX}_{\mathrm{x}} / \mathrm{PTX}_{\mathrm{o}}\right)$ from glass slides $(\phi=6 \mathrm{~mm}, n=4)$ coated with $3 \mu \mathrm{g} / \mathrm{mm}^{2}$ PTX, either embedded in $75 \mathrm{wt} \%$ PVP or free from matrix, in function of crosslinking time under UV light exposition at $254 \mathrm{~nm}$. Data shown as mean $\pm \mathrm{SD}$. Insert: Proposed radical formation mechanism of PTX leading to decomposition and/or crosslinking with PVP according to Chen et al. [26].

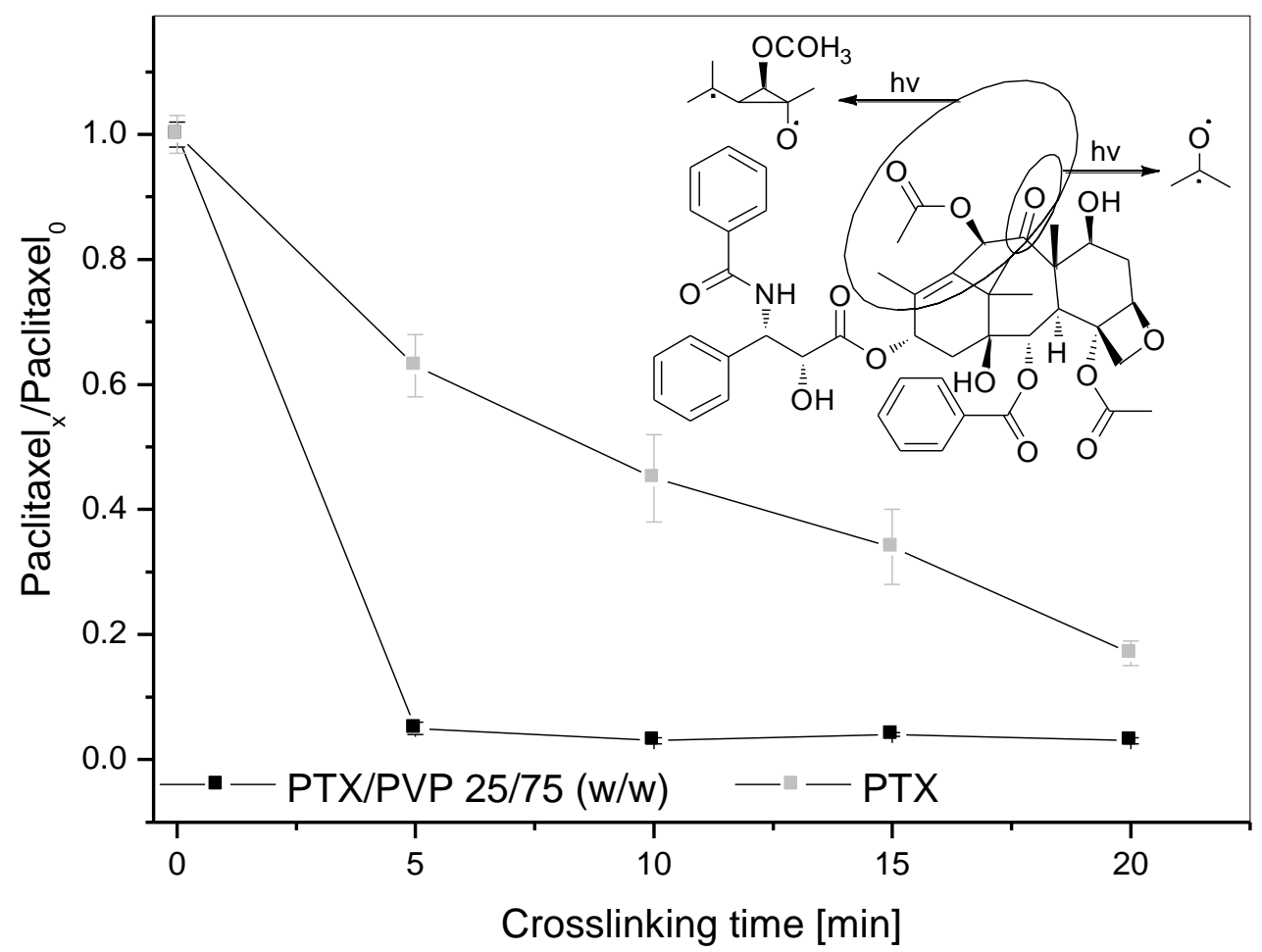


Figure 3. Representative electron micrographs of balloon catheter spray-coated with PVP, which has been crosslinked after coating via UV radiation at $254 \mathrm{~nm}$ for $10 \mathrm{~min},(\mathbf{a}, \mathbf{b})$ prior and (c,d) after 25 wt \% PTX incorporation via pipetting. Micrographs a and b evidence an intended coating boundary for better visualization of deposited PVP on the right.
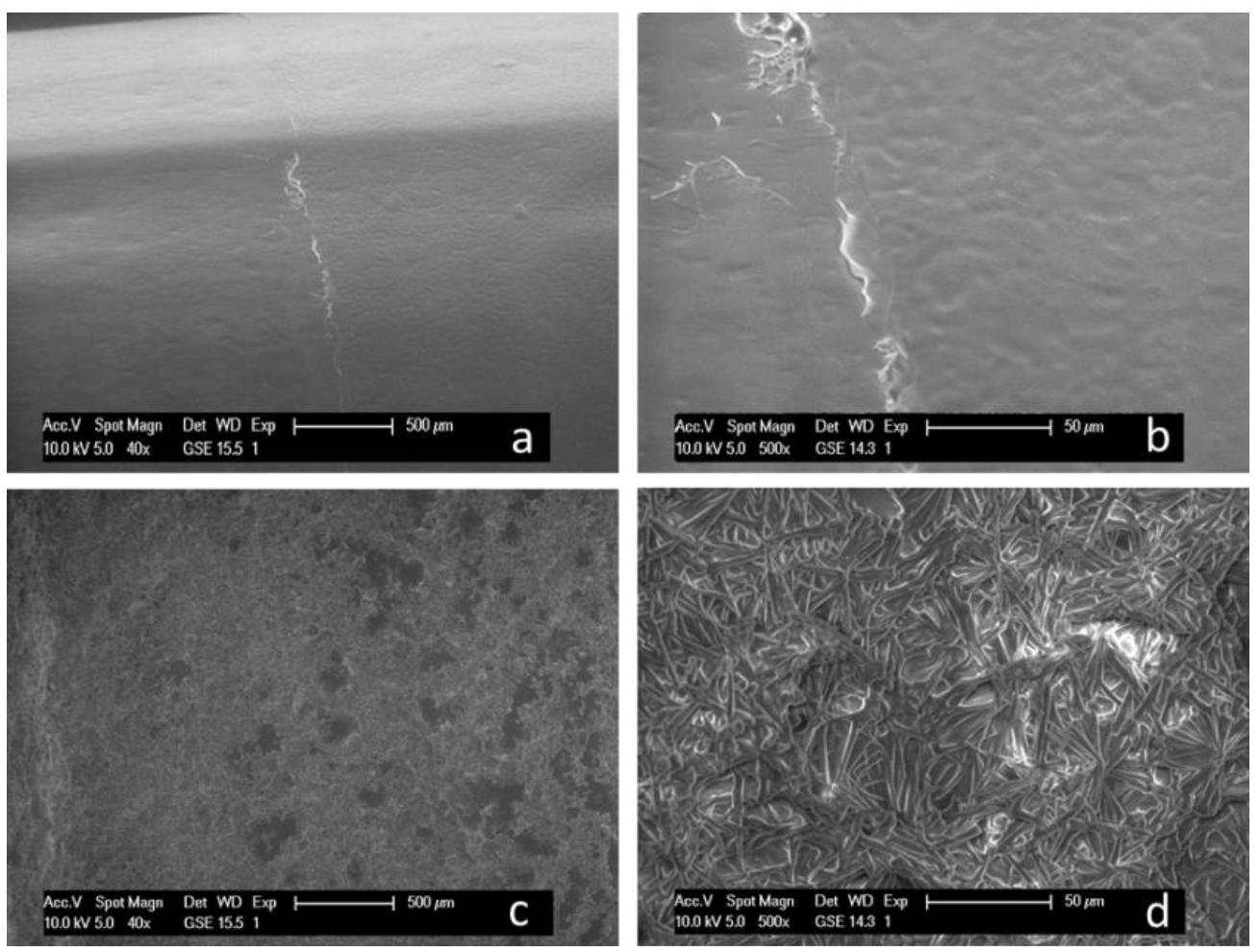

As we contrarily assume from our own and foreign previous investigations, that crosslinking density has an impact on drug release [28,29], resulting performance of photochemically crosslinked PVP coatings on DCB was again evaluated with regard to PTX wash off and transfer upon balloon expansion in a silicone tube as vessel model in dependence of UV radiation time. Figure 4 evidences observed tendencies, which were decreasing PTX wash off rates with elevated radiation time and initially increased PTX transfers upon balloon expansion in a silicone tube, with a maximum at 10 min radiation, followed by decay to the base level.

The decrease of the PTX wash off rate is probably associated with a reduction of the PVP water solubility with higher crosslinking density resulting in increased coating stability during $1 \mathrm{~min}$ immersion in PBS/Tween20. This assumption can be underlined by the observation of PVP coating masses prior and after contact with aqueous medium. While less than $50 \%$ of the coating mass was detected on glass slides without and after 5 min of UV radiation, higher radiation times allowed up to $100 \%$ mass retention meaning good coating stability after $1 \mathrm{~min}$ immersion in PBS/Tween20 (Figure 5a). Interestingly, more than 10 min of crosslinking were needed for 100\% PVP mass retention after 2 min of immersion. Representative photographs of PVP-coated glass slides evidence a progressive opacity of slides with longer UV radiation. By means of ESEM micrographs, we could attribute this observation to the enclosure of salts from buffered medium during immersion (Figure 5b,c), supporting the presence of the PVP coating even after immersion. As only the photograph of the glass slide, radiated for $10 \mathrm{~min}$, evidenced differences between 1 and $2 \mathrm{~min}$ of immersion, we did not display representative photographs of further glass slides after 2 min of immersion. 
Figure 4. Percental PTX wash off after one minute elution in DPBS/Tween20, PTX transfer upon expansion in silicone tube (30 s, 7 bar) and residual PTX load of DCB spray-coated with PVP containing $25 \mathrm{wt} \%$ PTX incorporated via pipetting at $23 \pm 2{ }^{\circ} \mathrm{C}$ in function of PVP crosslinking time under UV radiation at $254 \mathrm{~nm}$.

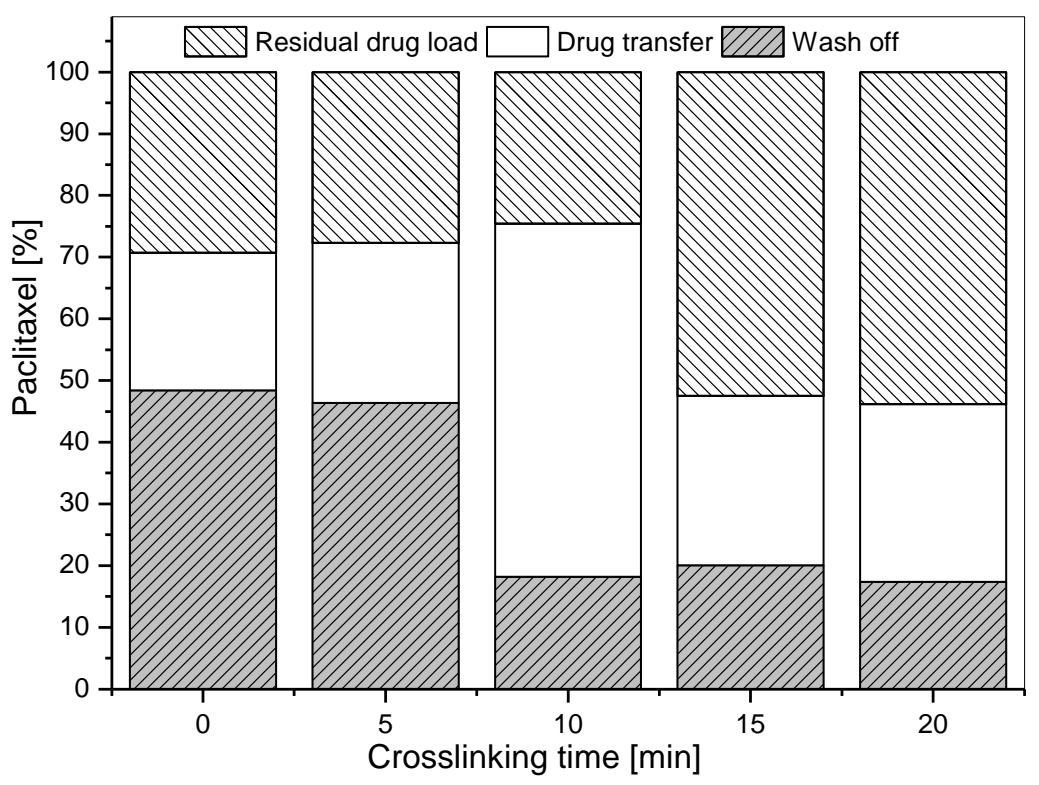

Figure 5. (a) Remaining mass of PVP coatings $\left(m_{\mathrm{PVP}(\mathrm{x})} / m_{\mathrm{PVP}(0)}\right)$ on glass slides $(\phi=6 \mathrm{~mm}$, $n=4$ ) after 1 and $2 \mathrm{~min}$ of immersion in $1 \mathrm{~mL}$ of DPBS/Tween 20 at $23 \pm 2{ }^{\circ} \mathrm{C}$ in function of crosslinking time $(0,5,10,15,20 \mathrm{~min})$ under UV radiation at $254 \mathrm{~nm}$. Data shown as mean \pm SD. Representative photographs of corresponding PVP coatings on glass slides are inserted within the graph. (b,c) ESEM micrographs of PVP coatings after $20 \mathrm{~min}$ crosslinking (b) prior and (c) after $1 \mathrm{~min}$ immersion in $1 \mathrm{~mL}$ DPBS/Tween 20 at $23 \pm 2{ }^{\circ} \mathrm{C}$.
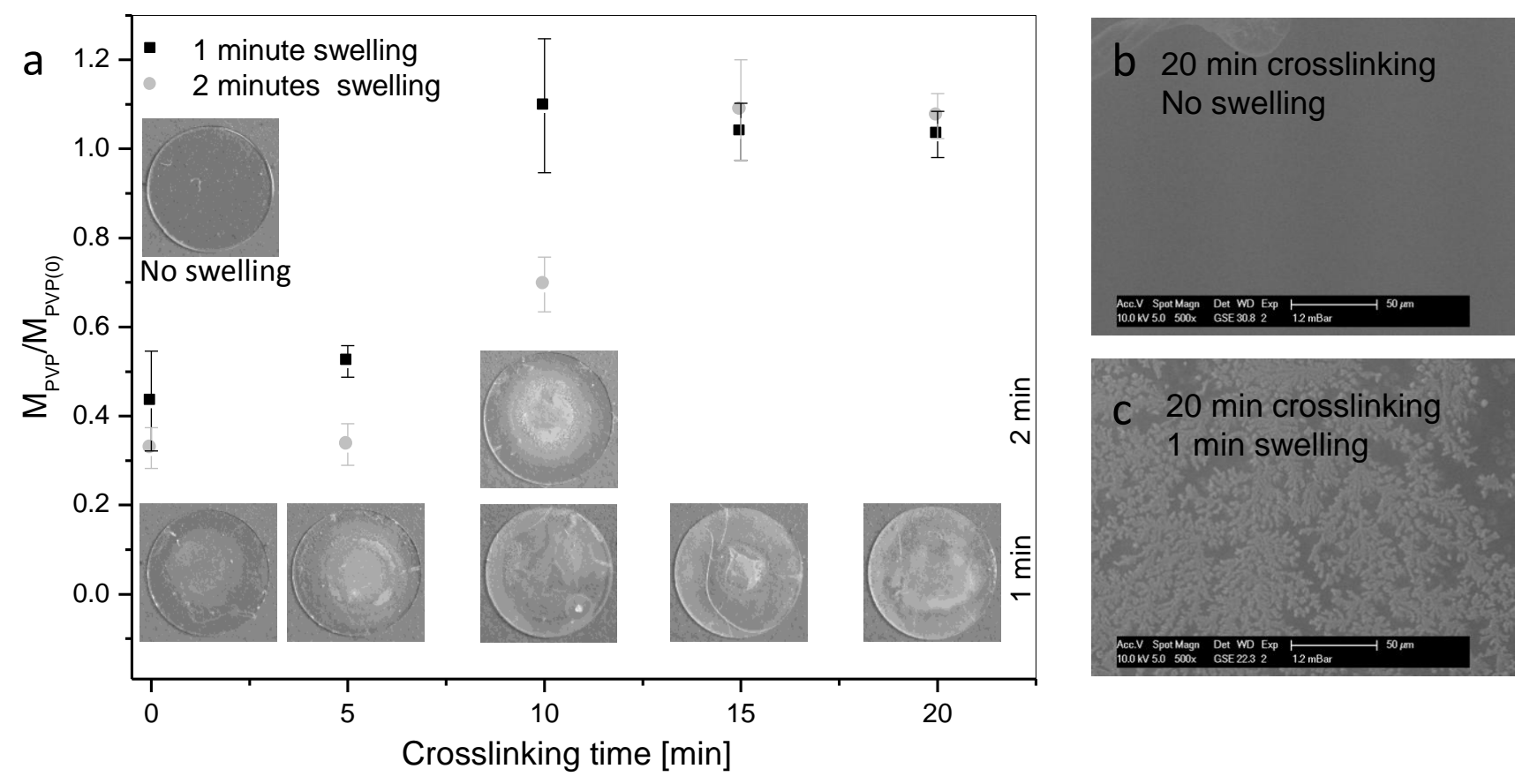

Crosslinking time [min] 
Interestingly, increased drug transfer was not directly correlated with decreased wash off but reached a maximum at 10 min crosslinking and decayed to the base level with longer radiation time, although wash off was not considerably changed. This seems to be associated with stability and water uptake of the PVP coating.

After 10 min radiation the PVP coating reached a maximum in water uptake after $1 \mathrm{~min}$ immersion in PBS/Tween20 (Table 1), which seems to favor drug transfer upon balloon expansion. Moreover, the instability of the coating with a longer immersion time might lead to separation of the hydrogel coating from the balloon surface upon pressing against the silicone tube and, thereby, support PTX transfer. Thus, crosslinking via 10 min UV radiation seems to be best suitable for the generation of PVP-based DCB coatings, as it results in sufficient coating stability to minimize drug wash off while still allowing high water uptake and efficient drug transfer upon balloon expansion.

Table 1. Water uptake of polyvinylpyrrolidone (PVP) coatings on glass slides $(n=4)$ after 1 and $2 \mathrm{~min}$ of immersion in $1 \mathrm{~mL}$ DPBS/Tween20 at $23 \pm 2{ }^{\circ} \mathrm{C}$. Data shown in $\mathrm{mg}$ water per mg initial coating mass as mean $\pm \mathrm{SD}$.

\begin{tabular}{ccc}
\hline Crosslinking time (min) & 1 min immersion & 2 min immersion \\
\hline 0 & $3.83 \pm 0.30$ & $3.65 \pm 0.46$ \\
5 & $3.47 \pm 0.41$ & $6.17 \pm 0.57$ \\
10 & $7.46 \pm 1.42$ & $12.22 \pm 4.36$ \\
15 & $6.85 \pm 1.74$ & $16.59 \pm 2.36$ \\
20 & $6.56 \pm 1.74$ & $16.53 \pm 1.29$ \\
\hline
\end{tabular}

\subsection{Simulated Use of PVP-Coated DCB}

In order to further evaluate the applicability of above optimized conditions for the generation of photochemically crosslinked PVP coatings for DCB, we simulated their use in an in vitro model. Therefore, DCB were manually advanced through a track model, consisting of a guiding catheter with a guide wire and a tortuous vessel path under measurement of drug wash off and transfer. Additionally, to the above-described experiments, this set-up should include mechanical strain on the coating during insertion of the catheter to the area of clinical interest. This inclusion of mechanical strain on the coating became apparent by a higher drug wash off. Thus, while we observed a drug wash off rate of $18 \%$ by simple immersion (Figure 4), insertion in the vessel model afforded a drug wash off rate of $34 \%$ (Figure 6). Drug wash off rates of non-crosslinked PVP coatings, of which use was simulated for comparison, were with $48 \%$ and $95 \%$ during immersion and simulated use, respectively (Figures 4 and 6), considerably higher. Interestingly, observed PTX wash off rates of photochemically crosslinked PVP-coatings are in the same range as above-mentioned PTX losses of 26\%-42\% reported in literature for balloons coated with urea or iopromide, respectively, during in vitro passage through a hemostatic valve and a guiding catheter $[11,12]$. Comparison to literature values should however be handled with care, as different in vitro models are involved. Regarding drug transfer upon balloon expansion, this comparison is even harder, as available literature values refer to PTX retention within native vessels after in vivo balloon angioplasty. For instance, Kelsch et al., and Scheller et al., reported a drug retention of 20\% of initial PTX balloon load of 15-20 min and of 17\% of initial PTX load of 40-60 min, post balloon dilatation, respectively [11,30]. As these studies dealt with DCB already efficiently and safely 
applied in clinical routine, the, here observed, $49 \%$ drug transfer for photochemically crosslinked PVP-based DCB during simulated use in vitro can be considered as a promising result.

Figure 6. Absolute PTX wash off during track, PTX transfer in silicone tube, residual and total PTX load of DCB spray-coated with PVP containing 25 wt \% PTX incorporated via pipetting after simulated use in track model according ASTM F2394-07, X.2.4. No PVP crosslinking or $10 \mathrm{~min}$ UV light exposition at $254 \mathrm{~nm}$ were applied prior to PTX incorporation. Bars show mean \pm SD of five experiments. Insert: Schematic pathway of the applied track model according ASTM F2394-07, X.2.4, added by guiding catheter and a silicone tube for balloon expansion.

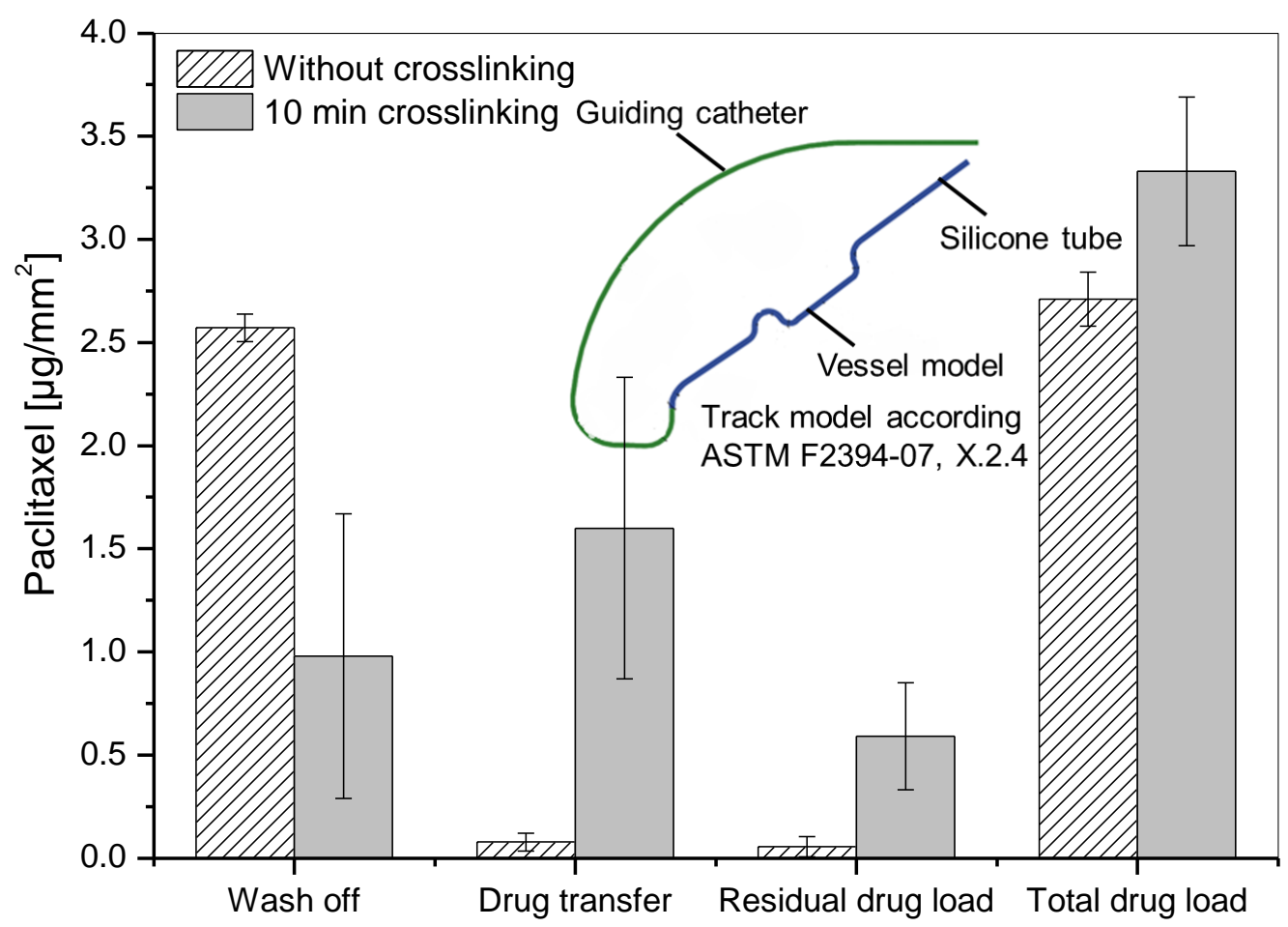

Moreover, in order to overcome lacking experimental set-up comparability, we additionally simulated the use of commercially available DCB with a urea-based coating, as we already reported previously [27]. With observed PTX drug transfer rates of $12 \%$, the high potential of PVP as coating matrix for DCB with low drug loss and efficient drug transfer could be successfully underlined. Nevertheless, further applicability of the presented coating design concerning mechanical performance characteristics, sterilizability, and storage stability, but, most notably, the in vivo safety and efficacy of the developed DCB still requires further research.

\section{Conclusions}

Within this study, we developed and thoroughly characterized a PTX containing PVP coating for DCB with regard to in vitro drug wash off and transfer upon expansion. Crosslinking of PVP evidenced essential for reduction of PTX wash off rates during insertion of the DCB to the vasculature and, thereby, for efficient drug transfer. The chosen inexpensive and biocompatible UV radiation process did, however, afford drug incorporation after the crosslinking process due to photodecomposition and/or 
involvement of PTX in vinylic polymerization with PVP, possibly lowering PTX bioactivity and availability. Moreover, we could show that radiation time has a considerable impact on performance of DCB. While short radiation times did not provide enough coating stability, associated with high wash off rates, long radiation times lowered drug transfer efficiency. The latter is possibly dedicated to lower water uptake with increasing crosslinking density and lacking separation of the hydrogel coating from the balloon surface upon compression of the coating during balloon expansion, which is supposed to support PTX transfer. Within our experimental set-up, PVP crosslinking via 10 min UV radiation seems to be most suitable for DCB, as minimized drug wash off could be combined with efficient drug transfer upon balloon expansion. Although correlation to values reported in literature for DCB, already efficiently and safely applied in clinical routine, is only possible to a limited extent due to varying experimental conditions, crosslinked PVP seems a very promising novel matrix for PTX-delivering angioplasty balloon catheter. In a previous study, we have proposed the use of the ionic liquid cetylpyridinium salicylate as additive for DCB with equally promising results [27]. However, PVP is in contrast to ionic liquids already established as hydrophilic lubricious coating on catheter-based cardiovascular devices in order to ease movement through the vasculature. The presented coating approach should be, hence, easily combinable with already commercially applied lubricious coating formulations by incorporating PTX directly within the via dip-coating deposited, photochemically-cured PVP coating, described by Babcock et al. [15]. Thus, no additional chemical reagent would be affordable to provide the device with an efficient local drug delivery function, lowering the risk of biocompatibility concerns and possibly simplifying the approval of the medical device later on.

\section{Acknowledgments}

The authors thank Dalibor Bajer, Thomas Reske, Martina Schröder and Christopher Schwerdt for their expert technical assistance and the Biotronik SE \& Co. KG, Erlangen, Germany for the generous supply of the uncoated balloon catheter. Furthermore, financial support from Bayer Material Science AG, Leverkusen, Germany and the Bundesministerium für Bildung und Forschung (BMBF) within REMEDIS "Höhere Lebensqualität durch neuartige Mikroimplantate" (FKZ: 03IS2081) is gratefully acknowledged.

\section{Conflicts of Interest}

The authors declare no conflict of interest.

\section{References}

1. Deloose, K.; Lauwers, K.; Callaert, J.; Maene, L.; Keirse, K.; Verbist, J.; Peeters, P.; Bosiers, M. Drug-eluting technologies in femoral artery lesions. J. Cardiovasc. Surg. 2013, 54, 217-224.

2. Buechel, R.; Stirnimann, A.; Zimmer, R.; Keo, H.; Groechenig, E. Drug-eluting stents and drug-coated balloons in peripheral artery disease. VASA 2012, 41, 248-261.

3. De Labriolle, A.; Pakala, R.; Bonello, L.; Lemesle, G.; Scheinowitz, M.; Waksman, R. Paclitaxel-eluting balloon: From bench to bed. Catheter. Cardiovasc. Interv. 2009, 73, 643-652. 
4. Waksman, R.; Pakala, R. Drug-eluting balloon: The comeback kid? Circ. Cardiovasc. Interv. 2009, 2, 352-358.

5. Baumbach, A.; Herdeg, C.; Kluge, M.; Oberhoff, M.; Lerch, M.; Haase, K.K.; Wolter, C.; Schröder, S.; Karsch, K.R. Local drug delivery: Impact of pressure, substance characteristics, and stenting on drug transfer into the arterial wall. Catheter. Cardiovasc. Interv. 1999, 47, 102-106.

6. Scheller, B.; Speck, U.; Romeike, B.; Schmitt, A.; Sovak, M.; Böhm, M.; Stoll, H.-P. Contrast media as carriers for local drug delivery successful inhibition of neointimal proliferation in the porcine coronary stent model. Eur. Heart. J. 2003, 24, 1462-1467.

7. Cremers, B.; Clever, Y.; Schaffner, S.; Speck, U.; Böhm, M.; Scheller, B. Treatment of coronary in-stent restenosis with a novel paclitaxel urea coated balloon. Minerva Cardioangiol. 2010, 58, 583-588.

8. Joner, M.; Byrne, R.A.; Lapointe, J.-M.; Radke, P.W.; Bayer, G.; Steigerwald, K.; Wittchow, E. Comparative assessment of drug-eluting balloons in an advanced porcine model of coronary restenosis. Thromb. Haemost. 2011, 105, 864-872.

9. Cortese, B.; Bertoletti, A. Paclitaxel coated balloons for coronary artery interventions: A comprehensive review of preclinical and clinical data. Int. J. Cardiol. 2012, 161, 4-12.

10. Sternberg, K.; Grabow, N.; Petersen, S.; Weitschies, W.; Harder, C.; Ince, H.; Kroemer, H.K.; Schmitz, K.-P. Advances in coronary stent technology-Active drug-loaded stent surfaces for prevention of restenosis and improvement of biocompatibility. Curr. Pharm. Biotechnol. 2013, 14, 76-90.

11. Kelsch, B.; Scheller, B.; Biedermann, M.; Clever, Y.P.; Schaffner, S.; Mahnkopf, D.; Speck, U.; Cremers, B. Dose response to Paclitaxel-coated balloon catheters in the porcine coronary overstretch and stent implantation model. Invest. Radiol. 2011, 46, 255-263.

12. Berg, M.C.; Kolodziej, H.; Cremers, B.; Gershony, G.; Speck, U. Drug-coated angioplasty balloon catheters: Coating compositions and methods. Adv. Eng. Mater. 2012, 14, B45-B50.

13. Kallmes, D.F.; McGraw, J.K.; Evans, A.J.; Mathis, J.M.; Hergenrother, R.W.; Jensen, M.E.; Cloft, H.J.; Lopes, M.; Dion, J.E. Thrombogenicity of hydrophilic and nonhydrophilic microcatheters and guiding catheters. AJNR Am. J. Neuroradiol. 1997, 18, 1243-1251.

14. Nagaoka, S.; Akashi, R. Low-friction hydrophilic surface for medical devices. Biomaterials 1990, $11,419-424$.

15. Babcock, D.E.; Hergenrother, R.W.; Craig, D.A.; Kolodgie, F.D.; Virmani, R. In vivo distribution of particulate matter from coated angioplasty balloon catheters. Biomaterials 2013, 34, 3196-3205.

16. Robinson, B.V. PVP: A Critical Review of the Kinetics and Toxicology of Polyvinylprrolidone (Povidone); Taylor \& Francis: London, UK, 1990.

17. Barros, J.A.G.; Fechine, G.J.M.; Alcantara, M.R.; Catalani, L.H. Poly(N-vinyl-2-pyrrolidone) hydrogels produced by Fenton reaction. Polymer 2006, 47, 8414-8419.

18. Rosiak, J.; Olejniczak, J.; Pȩkala, W. Fast reaction of irradiated polymers-I. Crosslinking and degradation of polyvinylpyrrolidone. Int. J. Radiat. Appl. Instrum. C 1990, 36, 747-755.

19. Henglein, A. Crosslinking of polymers in solution under the influence of gamma radiation. J. Phys. Chem. 1959, 63, 1852-1858.

20. Lopérgolo, L.C.; Lugão, A.B.; Catalani, L.H. Direct UV photocrosslinking of poly(N-vinyl-2-pyrrolidone) (PVP) to produce hydrogels. Polymer 2003, 44, 6217-6222. 
21. Speck, U.; Scheller, B.; Abramjuk, C.; Breitwieser, C.; Dobberstein, J.; Boehm, M.; Hamm, B. Neointima inhibition: Comparison of effectiveness of non-stent-based local drug delivery and a drug-eluting stent in porcine coronary arteries1. Radiology 2006, 240, 411-418.

22. Scheller, B.; Hehrlein, C.; Bocksch, W.; Rutsch, W.; Haghi, D.; Dietz, U.; Böhm, M.; Speck, U. Treatment of coronary in-stent restenosis with a paclitaxel-coated balloon catheter. N. Engl. J. Med. 2006, 355, 2113-2124.

23. Scheller, B.; Hehrlein, C.; Bocksch, W.; Rutsch, W.; Haghi, D.; Dietz, U.; Böhm, M.; Speck, U. Two year follow-up after treatment of coronary in-stent restenosis with a paclitaxel-coated balloon catheter. Clin. Res. Cardiol. 2008, 97, 773-781.

24. Schmidt, W.; Lanzer, P. Instrumentation. In Catheter-Based Cardiovascular Interventions; Lanzer, P., Ed.; Springer: Berlin/Heidelberg, Germany, 2013; pp. 445-472.

25. Tønnesen, H.H. The Photostability of Drugs and Drug Formulations; Taylor \& Francis: London, UK, 1996.

26. Chen, S.-H.; Farina, V.; Huang, S.; Gao, Q.; Golik, J.; Doyle, T.W. Studies on the photochemistry of taxol®. Tetrahedron 1994, 50, 8633-8650.

27. Petersen, S.; Kaule, S.; Stein, F.; Minrath, I.; Schmitz, K.-P.; Kragl, U.; Sternberg, K. Novel paclitaxel-coated angioplasty balloon catheter based on cetylpyridinium salicylate: Preparation, characterization and simulated use in an in vitro vessel model. Mater. Sci. Eng. C 2013, 33, 4244-4250.

28. Petersen, S.; Kaule, S.; Teske, M.; Minrath, I.; Schmitz, K.-P.; Sternberg, K. Development and in vitro characterization of hyaluronic acid-based coatings for implant-associated local drug delivery systems. J. Chem. 2013, 2013, 587875:1-587875:11.

29. Hoffman, A.S. Hydrogels for biomedical applications. Adv. Drug Deliv. Rev. 2012, 64, S18-S23.

30. Scheller, B.; Speck, U.; Abramjuk, C.; Bernhardt, U.; Böhm, M.; Nickenig, G. Paclitaxel balloon coating, a novel method for prevention and therapy of restenosis. Circulation 2004, 110, 810-814.

(C) 2013 by the authors; licensee MDPI, Basel, Switzerland. This article is an open access article distributed under the terms and conditions of the Creative Commons Attribution license (http://creativecommons.org/licenses/by/3.0/). 